\title{
THE IMPORTANCE OF SOLAR WHITE-LIGHT FLARES
}

\author{
DONALD F. NEIDIG \\ Air Force Geophysics Laboratory, National Solar Observatory/Sacramento Peak, National Optical \\ Astronomy Observatories*, Sunspot, NM 88349, U.S.A.
}

\begin{abstract}
The basic results of white-light flare (WLF) photometric and spectrographic observations are reviewed. WLFs represent the most extreme density conditions in solar optical flares and are similar to stellar flares in many respects. It is shown that WLFs originate in the low chromosphere and upper photosphere, and that their huge radiative losses remain difficult to explain within the context of known mechanisms of energy transport.
\end{abstract}

\section{Introduction}

Solar white-light flares (WLFs) are defined as the components of flares that are visible in optical continuum or integrated light. WLF emission appears as patches, waves, or ribbons, often containing smaller $(<3 \mathrm{arc} \mathrm{sec})$ bright kernels (see descriptions and additional references in Neidig and Cliver, 1983a; and Canfield et al., 1986). When observed with small aperture patrol telescopes at $\lambda<4000 \AA$, WLFs occur at a rate $\approx 15$ per year near solar maximum (Neidig and Cliver, 1983b). Basic properties of WLFs, including their associated emissions, morphology, apparent lack of polarization, and the solar active regions in which they occur, are summarized in a catalog of events observed since 1859 (Neidig and Cliver, 1983a). Except for the appearance of continuum, WLFs bear no particular morphological or spectral distinction, nor are their associated emissions at X-ray or radio wavelengths unusual in any respect. WLFs are, however, among the more energetic solar flares, and it has been shown that optical continuum becomes visible whenever the flare EUV or soft X-ray luminosity exceeds a relatively large threshold (McIntosh and Donnelly, 1972; Neidig and Cliver, 1983b). Thus optical continuum is probably present in all flares, but attains a detectable level of brightness in relatively few cases. This conclusion carries with it the corollary that WLFs are not fundamentally different from ordinary flares. Nevertheless, WLFs are of great importance in flare research because they are similar in many respects to stellar flares (cf. Worden, 1983) and because they represent the most extreme conditions attained in solar optical flares, thereby presenting a major challenge to atmospheric models and energy transport mechanisms. The following sections review the spectral characteristics, energetics, and physical conditions in WLFs; these topics then lead to

\footnotetext{
* Operated by the Association of Universities for Research in Astronomy, Inc., under contract with the National Science Foundation. Partial support for the National Solar Observatory is provided by the USAF under a Memorandum of Understanding with the NSF.
} 
a final discussion on the consequences of WLFs to mechanisms of energy transport in highly energetic fiares.

\section{Gross Spectral Characteristics and Energetics}

WLF photometric data are usually obtained from photographic images in broad (10-100 ̊) spectral bands at several different wavelengths. Ordinarily the spectral bands are chosen to avoid strong chromospheric flare lines. However, numerous narrow absorption lines formed in the photosphere and low chromosphere are not excluded from these broad bandpasses, and, although relatively few of these lines show emission in WLFs, the photometric data cannot in any case be considered to be pure continuum. Figure 1 shows the WLF contrast, $\left(I_{f}-I_{0}\right) / I_{0}$, where $I_{0}$ is the intensity of the solar background, for the brightest kernels in four events. The spectra are relatively flat for $\lambda>4000 \AA$, but show a marked increase at $\lambda<4000 \AA$ due, in part, to the reduced brightness of the solar background at short wavelengths. Nevertheless, in terms of absolute intensity $\left(I_{f}-I_{0}\right)$ the WLFs in Figure 1 still average approximately three times brighter at $3600 \AA$ than at $5000 \AA$. Thus solar WLFs are 'blue', as are stellar flares. The increase in brightness at short wavelengths is attributed to: (1) the presence of Balmer continuum in some cases, (2) the higher temperature of the flare relative to the quiet Sun, (3) the merging of Balmer lines near the Balmer limit (Donati-Falchi, Falciani, and

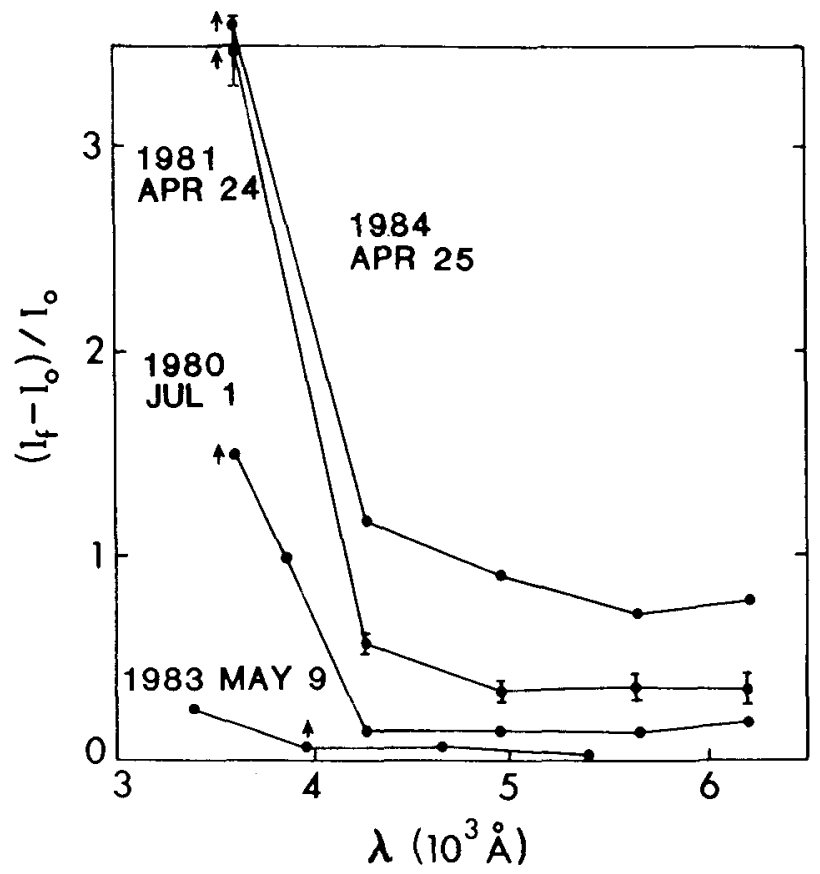

Fig. 1. Peak contrasts in the brightest kernels of four WLFs, measured in broad (20-50 $\AA$ ) bands. Arrows identify data points that are lower limits. The 1981 April 24 flare has the most reliable spectral shape. Except for the 1984 April 25 event, data are from Canfield et al. (1986). 
Smaldone, 1986), and (4) emission in photospheric and low chromospheric lines that become progressively more numerous at short wavelengths.

Peak surface fluxes, luminosities, and total energy radiated in the continuum of WLFs are summarized in Table I. These data assume that the WLF spectrum spans a range 2500-10 4 (Kane et al., 1985; Canfield et al., 1986). It should be noted that even the largest WLFs (Table I) have luminosities only $\approx 10^{-3}$ of the largest flares on dMe stars (cf. Gurzadyan, 1980). With regard to understanding the heating mechanisms of WLFs the most important datum is the peak flux, which, in the 1984 April 25 event, attained a value equivalent to a $100 \%$ enhancement over the quiet Sun. As shown in Section 4, the large fluxes observed in WLFs are not easily explained within the context of known mechanisms of flare energy transport.

TABLE I

Summary of WLF energetics

\begin{tabular}{llll} 
& $\begin{array}{l}\text { Flux in bright kernel } \\
\left(\mathrm{erg} \mathrm{s}^{-1} \mathrm{~cm}^{-2}\right)\end{array}$ & $\begin{array}{l}\text { Luminosity } \\
\left(\mathrm{erg} \mathrm{s}^{-1}\right)\end{array}$ & $\begin{array}{l}\text { Total energy } \\
(\mathrm{erg})\end{array}$ \\
\hline $\begin{array}{l}\text { Typical WLF } \\
\text { Largest WLF }\end{array}$ & $1-2 \times 10^{10}$ & $10^{27}-10^{28}$ & $10^{30}$ \\
$(1984$ April 25) & $6 \times 10^{10}$ & $2 \times 10^{29}$ & $>3 \times 10^{31}$ \\
\hline
\end{tabular}

a Data from Neidig and Cliver (1983a).

b Sacramento Peak data, not published elsewhere.

The ratio of the WLF continuum flux, $F_{\text {cont }}$, to the flux in emission lines at flare maximum may be similar to that found in stellar flares. For example, in the 1981 April 24 event (Neidig, 1983) $F_{\text {cont }} / F_{\mathbf{H} \alpha}=60$, which, after allowing a factor of 6 for the flux in emission lines other than $\mathrm{H} \alpha$, leads to the conclusion that approximately $90 \%$ of the total flux was in continuum - a value that compares well with stellar flares (Gurzadyan, 1980). However, due to the larger area and longer duration of the $\mathrm{H} \alpha$ emission the total energy radiated in $\mathrm{H} \alpha$ in the WLF can be comparable to that radiated in continuum (Slonim and Korobova, 1975).

\section{Physical Conditions Derived from Spectral Analysis}

Spectral analysis of WLFs is made difficult by (1) insufficient spatial and temporal resolution, (2) terrestrial atmospheric spectral dispersion, (3) improper placement of the spectrograph slit (the brightest kernels of WLFs have not yet been observed spectrographically!), and (4) inaccurate subtraction of the background solar spectrum (which is spatially irregular and often nearly as bright as the flare itself). In short, the relative nearness of the Sun has not afforded all possible advantages to WLF observations. Nevertheless, several important results have been obtained.

One significant discovery is the presence of Balmer continuum (Hiei, 1982; Neidig, 1983; Donati-Falchi, Falciani, and Smaldone, 1984; Neidig and Wiborg, 1984). The electron density, $N_{e}$, in the Balmer-emitting layers, as measured from Stark broadening 
of the high Balmer lines, was found to be $3-5 \times 10^{13} \mathrm{~cm}^{-3}$ for several bright events. Measurements of the optical thickness in the Balmer continuum and wings of low Balmer lines in the 1981 April 24 WLF (Neidig, 1983) yielded column densities of second-level hydrogen atoms $N_{2} z=1-3 \times 10^{16} \mathrm{~cm}^{-2}$. Usually, the line spectra of WLFs that show Balmer continuum are quite similar to spectra of bright non-WLFs; this suggests that the canonical temperature $\left(\approx 10^{4} \mathrm{~K}\right)$ of the optical flare applies also to WLFs, with the only essential difference being the somewhat larger values of $N_{e}$ and $\mathrm{N}_{2} z$. Allowing for uncertainties in flare temperature and departures from LTE, the geometric thickness, $z$, of the April $24 \mathrm{WLF}$ is probably $25-250 \mathrm{~km}$. The April 24 event also shows a Paschen jump (Neidig and Wiborg, 1984; absence of a Paschen jump in other WLFs has not been confirmed), indicating that the continuum at visible wavelengths in this flare is dominated by recombination $\left(H_{f b}\right)$ emission (thus retracting the original interpretation of $\mathrm{H}^{-}$emission by Neidig, 1983). This conclusion is corroborated by the observed absence of emission in the cores of metallic lines formed below $600 \mathrm{~km}$ in the atmosphere (Neidig, 1986a).

Two WLFs are known in which the Balmer continuum is apparently absent (Machado and Rust, 1974; Boyer et al., 1985); instead, the continuum in these events shows a monotonic increase toward short wavelengths. Machado and Rust (1974) attributed the continuum to $H_{f b}$ emission, although the absence of the Balmer jump presents a difficulty for this interpretation. In the event studied by Boyer et al. (1985) $H_{f b}$ emission was ruled out on the basis of the extremely weak Balmer lines; instead, the authors argued for $\mathbf{H}^{-}$ emission originating in a slightly warmed upper photosphere (evidence for strong heating near the quiet Sun $\tau_{5000}=1$ level has not been found in any WLF). Thermal bremsstrahlung from a hot $\left(T>10^{5} \mathrm{~K}\right)$ plasma seems unlikely in such events, as it is incompatible with observed fluxes in soft X-rays and EUV. Even temperatures in the range $2 \times 10^{4}-10^{5} \mathrm{~K}$ may not contribute significantly to the continuum, as helium lines in WLFs generally indicate small emission measures in this temperature range (Lites, Neidig, and Bueno, 1986).

Boyer et al. (1985) noted the possibility that the two, relatively faint WLFs without Balmer jumps - the spectrograms of which were obtained in the post-impulsive, or gradual, phase of the flare - might represent a phenomenon different from the bright continuum events associated with the impulsive phase (see also Machado and Rust, 1974; Rust, 1986). Machado et al. (1986) formally proposed the existence of two extreme types of WLF: Type I, showing strong Balmer lines and $H_{f}$ continuum originating in the chromosphere, and Type II, showing weak Balmer emission and $\mathrm{H}^{-}$continuum originating in the upper photosphere. Spectrograms of WLFs studied by Hiei (1982) and Hiei et al. (1982) would indicate a mixture of both types, as might be true of all WLFs. In this case, when the relative size of the Balmer jump and the temperature are known, it becomes possible to separate the contributions of $H_{f b}$ and $\mathrm{H}^{-}$emission at wavelengths longward of the Balmer jump if some assumption can be made on the flare optical thickness. In general, however, the relative size of the Balmer jump remains a function of the flare optical thickness when the condition $\tau_{\text {cont }} \ll 1$ does not hold (Grinin and Sobolev, 1977). This is an especially important point because large optical 


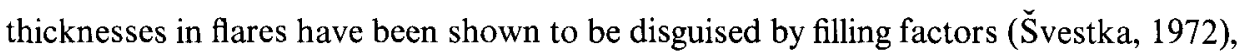
and the same is apparently true for WLFs as well (Neidig, 1986b).

Precise interpretation of the line and continuum spectra of WLFs requires selfconsistent models that can properly simulate the effects of unresolved structure as well as the distributions of mass, temperature, and velocity in the flare atmosphere. Unfortunately, the majority of available models (see references above) are very approximate, often assuming homogeneous, isothermal slabs. Even non-LTE, semi-empirical models (e.g., Avrett, Machado, and Kurucz, 1986; Gan and Fang, 1988), which are able to reproduce line and some continuum features of WLFs, presently suffer from the assumption of hydrostatic equilibrium, which effectively guarantees insufficient loading on the flare chromosphere to produce the emission measures necessary to explain bright continuum. High-pressure equilibrium, with $N T \geq 10^{18} \mathrm{~cm}^{-3} \mathrm{~K}$ everywhere within the flare loop above the photosphere, might be a more appropriate initial assumption for models.

\section{Energy Transport Mechanisms}

A conventional (albeit unproven) assumption of flare physics is that all of the flare optical emission is powered by energy released in the corona and subsequently transported to the high density $\left(N>10^{12} \mathrm{~cm}^{-3}\right)$ regions. WLFs are important in this context because their large radiative fluxes place severe constraints on known mechanisms of energy transport. Several transport mechanisms are summarized below.

\subsection{Heat CONDUCTION}

Assuming Spitzer conductivity and applying the analysis of Shmeleva and Syrovatskii (1973) under conditions of either constant density or constant pressure (the latter seems more likely to apply), it is found that, in order to sustain fluxes exceeding $10^{10} \mathrm{erg} \mathrm{s}^{-1} \mathrm{~cm}^{-2}$, the temperature gradient must be so steep that the emission measure at $T<10^{5} \mathrm{~K}$ will be too small to radiate the observed WLF flux. The predicted radiative flux in the constant pressure case is a factor $\approx 10^{3}$ too small. Heat conduction seems unlikely as a power source in stellar flares as well, unless the ratio of flare area to flare luminosity is much larger than in the solar case.

\subsection{Electron beAmS}

If solar flare hard X-ray bursts are interpreted according to a 'thick target' model (see Hudson, 1972; Švestka, 1976, and references therein), then the total power residing in nonthermal electrons with energies $E \geq 50 \mathrm{keV}$ has been shown in several cases to be sufficient to power WLFs in the chromosphere (Kane et al., 1985; Canfield et al., 1986). Electrons with $E \geq 50 \mathrm{keV}$ are thermalized in the solar atmosphere at densities exceeding $10^{13.5} \mathrm{~cm}^{-3}$, which is appropriate for chromospheric WLFs. Moreover, the time profiles of the hard X-ray and white light emissions are approximately similar (Rust and Hegwer, 1975; Canfield et al., 1986), and this appears to be true regardless of whether the emissions are impulsive or gradual in nature (Kane et al., 1985). The 1980 July 1 
WLF (Zirin and Neidig, 1981) is a possible exception to electron beam heating, as Canfield et al. (1986) have demonstrated that the column density over which the electrons deposited their energy was insufficient to produce the optical continuum. The latter calculation, however, did not make allowance for the full range of column densities appropriate to the range of electron energies that could power the WLF. Therefore, within the limits of various uncertainties, it may be premature to rule out electron beam heating in this WLF. On the other hand, the effects of reverse currents in reducing the effectiveness of beam heating at large densities have not been included in any of the calculations above. With regard to heating the upper photosphere during WLFs, electron beams are hopelessly inadequate, as electron energies $\geq 900 \mathrm{keV}$ are necessary for penetration to these depths, and the total power carried by these electrons is a factor $\geq 10^{2}$ too small.

A variation of the electron beam model (Livshits et al., 1981; MacNeice et al., 1984) proposes energy transport in two steps: explosive heating of the upper chromosphere by an electron beam (or any other source that produces explosive heating) generates a downward-propagating compression wave that heats the deeper atmosphere and produces the WLF. The expected time delay for the hydrodynamic response is on the order of $10 \mathrm{~s}$, which is not resolved in the WLF/hard X-ray profiles presented in Canfield et al. (1986).

\subsection{High-ENERGy ( $>4 \mathrm{MeV})$ PROTON BEAMS}

Quantitative analysis of gamma-ray and white light observations has been published for only one WLF (1980 July 1 (Ryan et al., 1983)), with the conclusion that thick target heating by high-energy protons was energetically insufficient at the time of WLF maximum. Ryan et al. (1983) concluded also that during the impulsive phase (which occurred well before the white light maximum was attained) the July 1 flare could have been powered by protons, but the same is true for electrons as well (Canfield et al., 1986). The July 1 event, although not spectrally or morphologically unusual, is the only known WLF whose time profile differs significantly from that of hard X-rays - a fact that might be noted when using this single case to rule out high-energy proton heating in general.

\subsection{LOW-ENERGY PROTONS}

Simnett (1986) proposed that low energy protons, accelerated by a series of small shocks, are the major energy carriers in the impulsive phase. These protons are stopped at relatively low density in the upper chromosphere, resulting in explosive heating that ultimately produces the WLF by a downward-propagating compression wave. A consequence of this model is that the hard X-rays are produced thermally. Starr et al. (1988) argue that thermally-produced hard X-ray bursts might be observationally difficult to distinguish from those produced by nonthermal electrons. Thus, aside from the disadvantage that low-energy protons produce no unique signature of their own, the low-energy proton/hydrodynamic model may suffer no observational contradictions; its evaluation as a transport mechanism in WLFs awaits quantitative predictions relating hard X-ray and white light emissions. 


\subsection{IRRADIATION BY $1-8 \AA \mathrm{X}$-RAYS}

At peak WLF luminosity the power in 1-8 $\mathrm{X}$-rays directed toward the solar surface is typically only $10^{-1}$ of the power radiated by the WLF, and in no case has the 1-8 $\AA$ power equaled or exceeded the peak WLF luminosity. This includes even the $1980 \mathrm{July} 1$ WLF which peaked nearly simultaneously with the soft X-ray event. Ordinarily, the $1-8 \AA \mathrm{X}$-rays peak $1-2$ minutes after the WLF maximum.

\subsection{IRRADIATION BY 10-1030 ̊̊ (EUV) EMISSION}

WLFs are associated with large EUV fluxes (McIntosh and Donnelly, 1972), corresponding to EUV luminosities in the range $6-23 \times 10^{27} \mathrm{erg} \mathrm{s}^{-1}$. Furthermore, it follows from the known temporal correlations between the WLF and hard X-rays and between hard X-rays and EUV, that the timing of the WLF and EUV emission must be similar as well. It can be speculated, therefore, that strong EUV emission (generated by an electron beam or other energy source in the upper chromosphere) might irradiate the deeper layers of the atmosphere and produce the WLF. A fundamental question, however, is whether EUV photons can attain sufficient range in an ambient atmosphere that is ordinarily opaque to most EUV radiation. This difficulty has been expressed recently by Poland, Milkey, and Thompson (1988).

\subsection{ALFVEN WAVES}

The dissipation of Alfvén waves in regions of high resistivity was proposed by Emslie and Sturrock (1982) as a means of explaining the warming of the temperature minimum region in ordinary flares (cf. Machado, Emslie, and Brown, 1978). The calculations of Emslie and Sturrock (1982) were aimed at energy deposition rates $\approx 10 \mathrm{erg} \mathrm{s}^{-1} \mathrm{~cm}^{-3}$, and made no attempt to account for rates $\approx 10^{3} \mathrm{erg} \mathrm{s}^{-1} \mathrm{~cm}^{-3}$ as would apply in WLFs. Delays of several seconds or more between the hard X-ray emission and the WLF are expected as a result of the finite velocity of the waves.

\section{Conclusions}

A wide variety of observational data and modeling indicates that (1) WLFs are not fundamentally different from ordinary flares, (2) WLFs are located in the lower chromosphere and upper photosphere, with no evidence for strong heating near the quiet Sun $\tau=1$ level, (3) the WLF light curve approximately follows the hard $(\approx 50 \mathrm{keV})$ $\mathrm{X}$-ray emission regardless of whether the hard $\mathrm{X}$-rays are associated with the impulsive or gradual phase of the flare, and (4) heat conduction, irradiation by soft X-rays, and heating by high-energy protons are not sufficient to power the WLF at the time of its peak luminosity. Heating by an electron beam, which might be important in chromospheric WLFs, is totally inadequate for the upper photospheric component of WLFs.

WLFs show considerable similarity to stellar flares, with one notable exception being the energetics. If solar and stellar flares can be described by models that are qualitatively similar, then the greater luminosity and total energy of stellar flares must ultimately 
derive from larger magnetic field energies and faster rates of conversion of the field energy into other forms. Observationally, these questions are probably less accessible than the simpler problem of understanding how the stellar flare atmosphere can sustain optical radiative losses as much as $10^{3}$ times greater than the largest solar WLFs. In answer to the latter it is speculated that, since both solar and stellar flares have temperatures that are nearly optimum $\left(\approx 10^{4} \mathrm{~K}\right)$ for optical radiative loss, the greater luminosity of stellar flares can be understood only in terms of larger flare area and greater optical thickness than in the solar case.

WLFs are astrophysically important because they challenge our understanding of flare energy transport, and because they illustrate that assumptions of hydrostatic equilibrium are probably inappropriate for flare models. They also remind us that, even in a star as near as the Sun, some flare structures remain unresolved, with the consequence that physical conditions derived from spectral analysis warrant careful interpretation. Finally, observed similarities between WLFs and stellar flares point to the universal nature of the flare phenomenon, and suggest that much can be gained from the study of each.

\section{References}

Avrett, E. H., Machado, M. E., and Kurucz, R. L.: 1986, in D. Neidig (ed.), The Lower Atmosphere of Solar Flares, National Solar Observatory, p. 216.

Boyer, R., Machado, M. E., Rust, D. M., and Sotirovski, P.: 1985, Solar Phys. 98, 255.

Canfield, R. C., Bely-Dubau, F., Brown, J. C., Dulk, G. A., Emslie, A. G., Enome, S., Gabriel, A. H., Kundu, M. R., Melrose, D., Neidig, D. F., Ohki, K., Petrosian, V., Poland, A., Rieger, E., Tanaka, K., and Zirin, H.: 1986, in M. Kundu and B. Woodgate (eds.), Energetic Phenomena on the Sun, NASA CP 2439, Chapter 3.

Donati-Falchi, A., Falciani, R., and Smaldone, L. A.: 1984, Astron. Astrophys. 131, 256.

Donati-Falchi, A., Falciani, R., and Smaldone, L. A.: 1986, in D. Neidig (ed.), The Lower Atmosphere of Solar Flares, National Solar Observatory, p. 136.

Emslie, A. G. and Sturrock, P. A.: 1982, Solar Phys. 80, 99.

Gan, W.-Q. and Fang, C.: 1988, Chin. Astron. Astrophys. 12, 146.

Grinin, V. P. and Sobolev, V. V.: 1977, Astrofizika 13, 587.

Gurzadyan, G. A.: 1980, Flare Stars, Pergamon Press, New York.

Hiei, E.: 1982, Solar Phys. 80, 113.

Hiei, E., Tanaka, K., Watanabe, T., and Akita, K.: 1982, Hinotori Symposium on Solar Flares, Institute of Space and Astronautical Science, Tokyo, p. 208.

Hudson, H. S.: 1972, Solar Phys. 24, 414.

Kane, S. R., Love, J. J., Neidig, D. F., and Cliver, E. W.: 1985, Astrophys. J. 290, L45.

Lites, B. W., Neidig, D. F., and Trujillo Bueno, J.: 1986, in D. Neidig (ed.), The Lower Atmosphere of Solar Flares, National Solar Observatory, p. 101.

Livshits, M. A., Badalyan, O. G., Kosovichev, A. G., and Katsova, M. M.: 1981, Solar Phys. 73, 269.

Machado, M. E. and Rust, D. M.: 1974, Solar Phys. 38, 499.

Machado, M. E., Emslie, A. G., and Brown, J. C.: 1978, Solar Phys. 58, 363.

Machado, M. E., Avrett, E. H., Falciani, R., Fang, C., Gesztelyi, L., Hénoux, J.-C., Hiei, E., Neidig, D. F., Rust, D. M., Sotirovski, P., Śvestka, Z., and Zirin, H.: 1986, in D. Neidig (ed.), The Lower Atmosphere of Solar Flares, National Solar Observatory, p. 483.

MacNeice, P., McWhirter, R. W. P., Spicer, D. S., and Burgess, A.: 1984, Solar Phys. 90, 357.

McIntosh, P. S. and Donnelly, R. F.: 1972, Solar Phys. 23, 444.

Neidig, D. F.: 1983, Solar Phys. 85, 285. 
Neidig, D. F.: 1986a, in D. Neidig (ed.), The Lower Atmosphere of Solar Flares, National Solar Observatory, p. 142.

Neidig, D. F.: 1986b, in D. Neidig (ed.), The Lower Atmosphere of Solar Flares, National Solar Observatory, p. 152.

Neidig, D. F. and Cliver, E. W.: 1983a, Air Force Geophysics Laboratory Technical Report AFGL-TR-83-0257, Hanscom AFB, Mass.

Neidig, D. F. and Cliver, E. W.: 1983b, Solar Phys. 88, 275.

Neidig, D. F. and Wiborg, P. H.: 1984, Solar Phys. 92, 217.

Poland, A. I., Milkey, R. W., and Thompson, W. T.: 1988, Solar Phys. 115, 277.

Rust, D. M.: 1986, in D. Neidig (ed.), The Lower Atmosphere of Solar Flares, National Solar Observatory, p. 282.

Rust, D. M. and Hegwer, F.: 1975, Solar Phys. 40, 141.

Ryan, J. M., Chupp, E. L., Forrest, D. J., Matz, S. M., Rieger, E., Reppin, E., Kanbach, G., and Share, G. H.: 1983, Astrophys. J. 272, L61.

Shmeleva, O. P. and Syrovatskii, S. I.: 1973, Solar Phys. 33, 341.

Simnett, G. M.: 1986, Solar Phys. 106, 165.

Slonim, Y. M. and Korobova, Z. B.: 1975, Solar Phys. 40, 397.

Starr, R., Heindl, W. A., Crannell, C. J., Thomas, R. J., Batchelor, D. A., and Magun, A.: 1988, Astrophys. J. 329, 967.

Švestka, Z.: 1972, Solar Phys. 24, 154.

Švestka, Z.: 1976, Solar Flares, D. Reidel Publ. Co., Dordrecht, Holland.

Worden, S. P.: 1983, in P. B. Byrne and M. Rodono (eds.), Activity in Red Dwarf Stars, D. Reidel Publ. Co., Dordrecht, Holland, p. 207.

Zirin, H. and Neidig, D. F.: 1981, Astrophys. J. 248, L45. 